\title{
An Interactive Modeling Method of 3D Model Based on National Cultural Resource Database
}

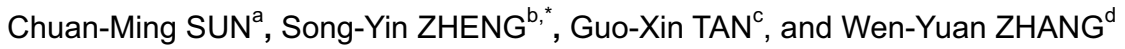 \\ National Research Center of Cultural Industries, Central China Normal University, Wuhan 430079, China \\ ${ }^{a} c{ }^{@}$ mail.ccnu.edu.cn, ${ }^{b}$ zhengsongyin@126.com' ${ }^{c}$ gxtan@mail.ccnu.edu.cn, ${ }^{d}$ wenyuanzhang@126.com \\ ${ }^{*}$ Corresponding author: zhengsongyin@126.com
}

\begin{abstract}
Aiming at solving the problems of low efficiency and complicated operation of 3D modeling in the process of virtual product development of national culture, this paper discusses a synthesis approach of surface geometry model based on data-driven. Firstly, a shape-based matching algorithm is used to retrieve the corresponding components in the database. Secondly, a new model will be generated through segmenting and assembling these components. Finally, this approach is applied to construct 3D model of scenes and characters in ancient Badong town. The application shows that this approach can generate the geometric model of characters and scenes efficiently and economically in cultural activities.
\end{abstract}

\section{Introduction}

Virtual 3D reconstructions of destroyed or disappeared cultural heritage can display the cultural activities to viewers effectively. For example, Selma Rizvić present their work on interactive virtual cultural heritage applications with storytelling and show how users appreciate this presentation form, considering it as breathing life into 3D geometry[1].Athanasios Voulodimos presents a system designed and developed for the 3D reconstruction of cultural heritage assets based on photogrammetry and clustering[2]. But these approaches also either need a large amount of time to construct the database or have more complicated steps.

This paper discusses a modeling method of data-driven geometry model by reassembling the components of existing 3D models in the resource database to create new models. First of all, a component matching algorithms in database is designed. Second, an optimization algorithm for surface model alignment is used. Third, an intelligent grid cutting algorithm is discussed. Finally, based on the 3D model data of the cultural resource database, this approach is applied to construct the 3D model of scenes and characters in ancient Badong town which was existed in Song dynasty.

\section{Modeling Method of 3D Model Based on Resource Database}

In the modeling of virtual cultural products, the most important problem is the construction of realistic virtual scene and role model. In this paper, the $3 \mathrm{D}$ modeling method is divided into three categories, for example, primitive construction method, model reconstruction method and sample interpolation synthesis method[3]. The method of primitive construction mainly adopts the traditional modeling software (Maya, 3Ds Max, etc.) for interactive editing of $3 \mathrm{D}$ model, which requires creators to have higher creativity and use software expertly. As the result, the efficiency is not high; Model reconstruction methods focus on a certain information based on a particular human body or scene for geometric reconstruction, information sources include photos, video, 3D scanning, which requires higher information of shape information. The sample interpolation synthesis method needs to construct the sample database before, and then realize parameterized feature of the reconstructed 3D model. Finally, the 3D model can be generated by mapping the characteristic parameters to the model geometry. This method has high modeling efficiency, but the structure of the sample database is complicated. By the geographical, historical and cultural factors, there is a big difference in modeling of characters and activity scenes in different cultural activities. In order to realize the rapid and efficient modeling of the virtual product model of the national culture, a 3D model modeling method is designed based on the national cultural resource database, which integrates the 3D model geometric representation and modeling method. 


\subsection{Component Retrieval}

The retrieval methods of 3D model can be divided into text-based retrieval and content-based retrieval. Text-based retrieval has matured, but the text is difficult to fully describe the complex 3D model. Content-based retrieval is automatic retrieval based on the actual content (geometry, topology, etc.) of a 3D model. The core is to extract a set of features to represent the $3 \mathrm{D}$ model, and then to retrieve the model by comparing its eigenvalues[4]. The existing algorithms of this method can be divided into three categories:(1)shape-based retrieval techniques, such as the shape distribution algorithm proposed by Osada[5]; (2) topological structure-based retrieval techniques, such as Hilaga's Reeb graph topology matching method; (3) Based on the comparison of image retrieval technology, such as
2D contour comparison method based on 3D model proposed by Funkhouser[6] .

The 3D models of national culture need higher shape fidelity, Osada's shape distribution method is used in this section. That is, by calculating the probability distribution of the model data, a description of the shape distribution of the models which are obtained. Since the shape distribution method is based on a large number of statistical data, it can satisfy the scaling invariant, rotation invariant, translation invariant and symmetric invariant, and is robust to noise, resampling and simplification. Using a large number of samples, the Euclidean distance of any two points on the surface of the 3D model is calculated (as shown in Fig.1 for a cross-sectional distance of a column model). Using these data, the model features can be described by the shape distribution data.

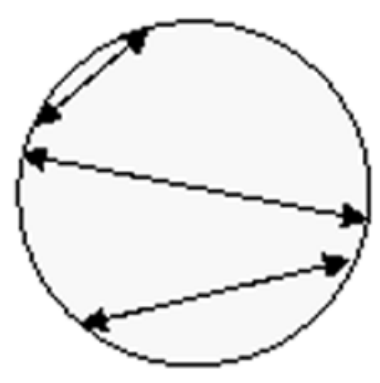

Figure 1. D2 distance of column cross-section

In this section, $\mathrm{f}$ and $\mathrm{g}$ are used to express the probability distributions of the two models respectively, $\hat{f}$ and $\hat{g}$ are used to express the distribution functions of the two models, $\hat{f}(x)=\int_{-\infty}^{x} f \hat{g}(x)=\int_{-\infty}^{x} g$.

Then the Minkowski L $\mathrm{N}$ distance of the probability distribution of the two models is $D(f, g)=\left(\int|f-g|^{N}\right)^{1 / N}, N=0,1, \infty$; The Minkowski LN distance of the distribution function is $D(f, g)=\left(\int|\hat{f}-\hat{g}|^{N}\right)^{1 / N}, N=0,1, \infty$ and similar model matching is performed by comparing the distance.

\subsection{Component Segmentation}

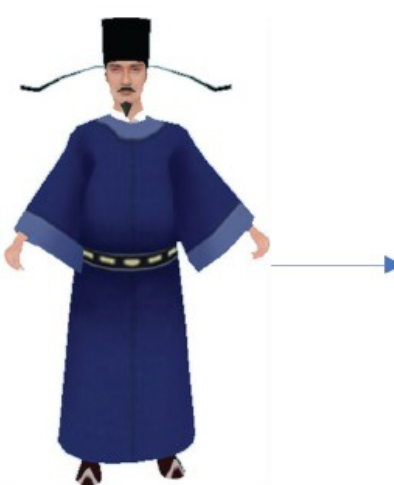

a The model to be split

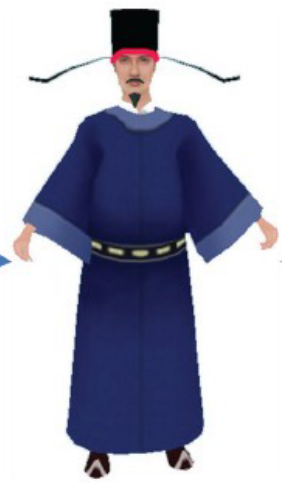

b Mark line

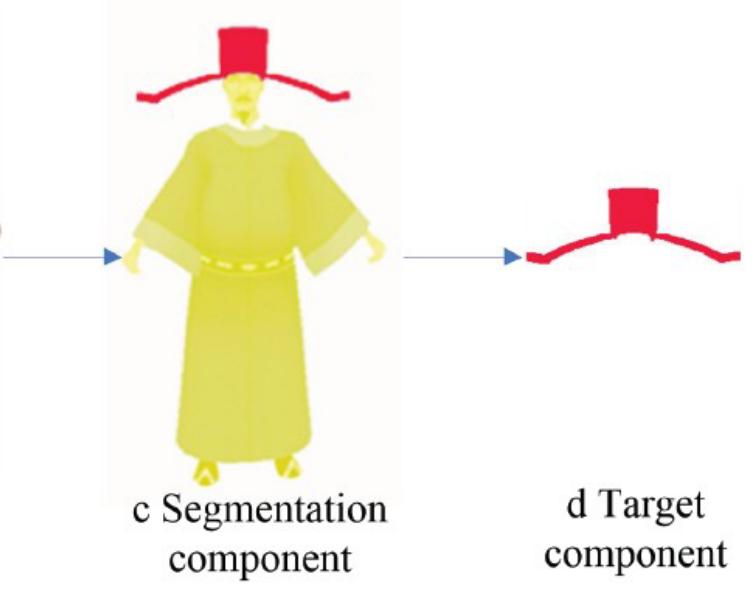

The components are sub-blocks of 3D models. There are many different method to display $3 \mathrm{D}$ models, and grid have become an important representations of $3 \mathrm{D}$ models [7]. At present, there are some 3D grid segmentation algorithms include watershed algorithm, grid representation based on entity representation, grid segmentation based on topology information and hierarchical decomposition based on fuzzy clustering. The interactive segmentation method can adjust the model segmentation in real time and improve the success rate of component acquisition. The existing 3D model interactive segmentation methods include: segmentation of 3D models using commercial modeling tools. By selecting the sequence vertices on the model surface grid and segmenting the model along the shortest path between them. 
Figure 2. Segmentation of 3D Model

According to the interactive segmentation method based on minimum cut in image domain, a simple and efficient interactive segmentation method is proposed by Zhao Mingxi[8]. First of all, the user only need to enter two groups or one group of mark lines on the screen which represent the background or objects, the system imposed some constraints. Then, a minimal cut algorithm with hard constraints is used to obtain the initial segmentation result on the simplified grid. Finally, the results are mapped back to the original grid, using the global optimal minimum cut algorithm to smooth the split results. It contains three steps which are shown in Fig.2:

(1)Grid simplification: This step is only used for the grid which has millinons of triangles. The first step is to simplify the grid. Because the quadratic grid simplification algorithm have features of good algorithm complexity and feature preserving, this algorithm is used to simplify the grid with large amount of data.

(2) Segmentation from the simplified grid: Using a screen-based interactive interface, users can easily specify a representative triangle on a simplified grid. Using these representative triangles as hard constraints, using this algorithm to find the best segmentation. For the results of the segmentation, the user can continue to adjust the results by adding triangles.

(3) Smooth cutting: the cutting edge is usually very rough when mapping cutting results from the simplified grid back to the original grid. On this basis, the traditional minimum cut algorithm will be used to smooth the cutting results.

\subsection{Component Assembly}

After getting the model component, how to automatically assemble into a new model is also an urgent problem to be solved. This paper has developed an interactive tool that allows the user to select and fix components which can be assembled to the new model, and then process the interface cracks of the assembled model surface. There are two difficult problems to be solved: components replacement and components seamless bonding.

The first problem is how to replace components which are existed in model. For example, using the component model which are retrieved form the resource database can replace the corresponding component of an existing model. It is necessary to provide an automatic command to fix the two corresponding components in the same coordinate system. This procedure is to achieve the invariant of the translation, the zoom, the rotation and the symmetry, and to achieve the minimum square sum of the distance between the each point of component surface and the nearest point of the other component. In this paper, by moving the centroid of the components to make it coincide with the centroid of the target component, the best translation can be achieved. The optimal scaling is achieved by changing the size ratio of the source components so that the mean variance (square sum of distances from each point to the centroid) is equal to the mean variance of the target component. Optimized rotation requires the use of Horn's method to establish point-to-point correspondence. ICP algorithm is a commonly method to calibrate two models, which converges a local optimal solution to minimize the sum of the squared-distance of the two models.

When assembling components into the model, it is difficult to create a seamless, smooth surface between the interface. This paper provides a simple method which was proposed by Kanai to connect these components seamlessly. This method is shown as follows: When connecting the two components, the user should select an open contour C1, C2 in each 3D grid model boundary (Fig.3(a)). When the "join" command is executed, the heuristic algorithm is used to automatically chamfer and mix, and achieve the vertex correspondence. That is, firstly, finding a pair of closest vertices $\mathrm{V} 1$ and $\mathrm{V} 2$ on $\mathrm{C} 1, \mathrm{C} 2$, as shown in Fig.3(b). And then, for $\mathrm{C} 2$, determining the positioning of $\mathrm{C} 1$ by checking the vector dot product from V1 to V1', the same method to define the vector from V2 to V2'. If the dot product is negative, then the positioning of $\mathrm{C} 1$ need be changed. Then, through the greedy algorithm iterations increment the current vertex on $\mathrm{C} 1$ or $\mathrm{C} 2$, picking a point where the Euclidean distance is the smallest in the current two vertices, and the corresponding vertex will be established. Finally, according to the corresponding vertex, the chamfer boundary is constructed (Fig.3(c)). In addition, the join command can smooth these nodes within the user-specified chamfer distance and the number of iterations by averaging the positions of the vertices and other neighbors. And then, a combined model (Fig.3(d)) with no traces is assembled. 


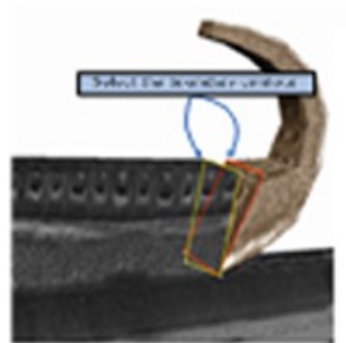

(a)

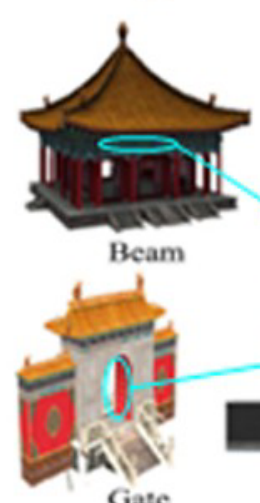

Gate

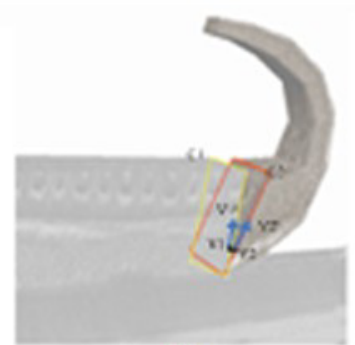

(b)

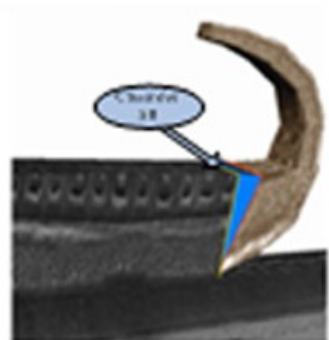

(c)

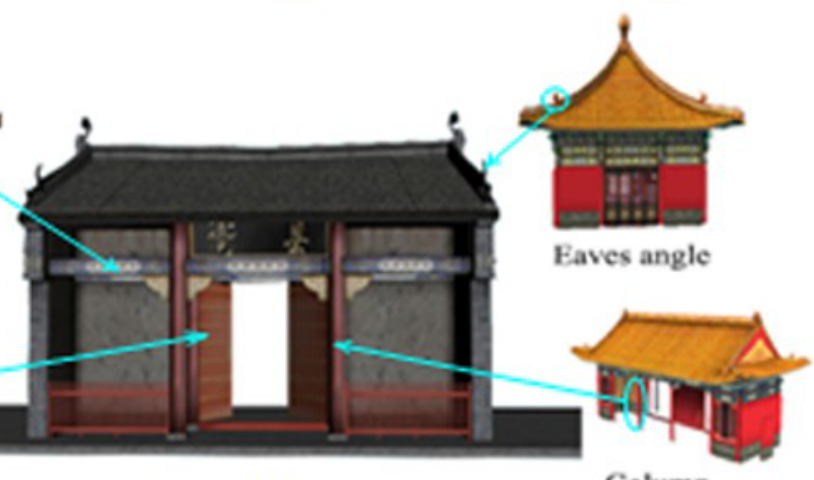

(d)

Figure 3. Assembly of 3D Model

\section{Experiment}

The three gorges dam is a huge project and the biggest hydropower station in china. When this dam project finished, many areas inclulde ancient cultural relic were submerged under water. As one of the key projects in the Three Gorges area, Archaeologists found the relics of old Badong town which was existed in Northern Song Dynasty, inluding official district, warehouse area, residential area, business district and so on,. Based on the national culture resource database, the models can be retrieved for intelligent segmentation to access the target components. These components can be combined into a variety of architectural and role models that can be displayed in a virtual environment. Finally, though the application of 3D geographic information system and VR technology, a experimental system, virtual Badong, is developed to rebuild these historic Badong town in the computer and display it from internet which is shown in Fig.4.

As Badong town is the ancient distribution center of immigrants, it also has very rich cultural. "Sayerhe" folk dance is an ancient funeral dance in this area. Through the deep research of the cultural activities and clothing in ancient Badong town, we bind the dance motion to the corresponding role model, and use motion data to drive the role model for cultural activities. In the virtual Badong, "sayerhe" folk dance is rebuilt and displayed online which is shown in Fig.5. 


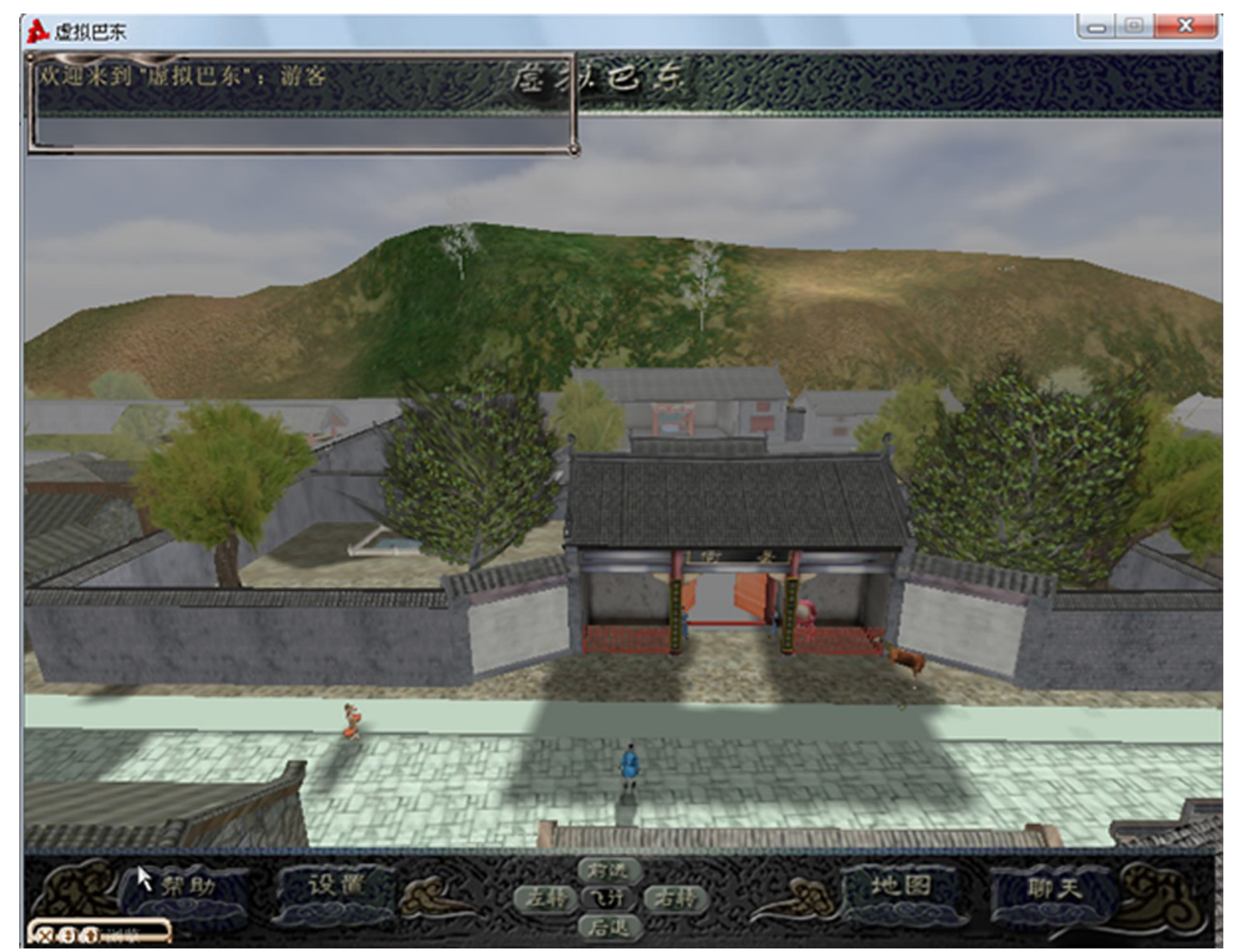

Figure 4. Government of Badong town

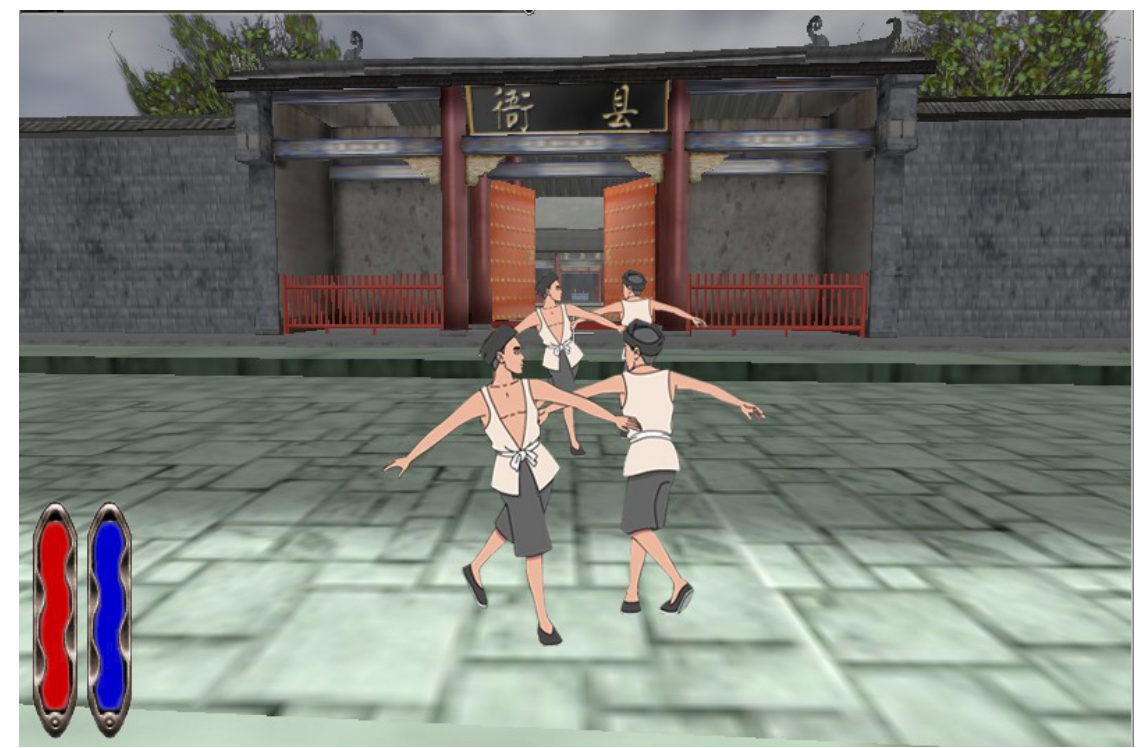

Figure 5. "sayerhe" folk dance

\section{Summary}

To sum up, a modeling method for 3D model based on resource database is proposed to rebuild the $3 \mathrm{D}$ scene and role modeling conveniently. Based on the analysis of the geometric representation and modeling method of the $3 \mathrm{D}$ model, the modeling method is used to generate the virtual scene and the role model in the cultural activities quickly and efficiently. Finally, based on archaeological data and the national cultural resource database, the 3D scene model and related characters are 
reconstructed, and the digital "sayerhe" folk dance also is displayed in this system. The application of "virtual Badong" prove that this method can be used for the representation of historical geography scenes and the protection of intangible cultural heritage.

\section{Acknowledgement}

This research was supported by the Research Funds for the Central Universities "Research on Knowledge visualization of traditional folk dance" and project of Scientific and Technical Supporting Programs of Hubei(NO. 2014BAA156).

\section{References}

1. Selma Rizvić, How to Breathe Life into Cultural Heritage 3D Reconstructions, European Review, 25(1) February 2017, pp. 39-50.

2. Athanasios Voulodimos; Nikolaos Doulamis, et al. Four-dimensional reconstruction of cultural heritage sites based on photogrammetry and clustering, Journal of Electronic Imaging, 26(1), 011013 (Nov, 2016).

3. $\mathrm{Xu}$ Aiguo. Research on 3D Garment Simulation Technology in Virtual Human Animation[D]. Zhejiang Hangzhou: Zhejiang University, 2006.

4. YANG Yu-bin, LIN Hui, ZHU Qing.Research on Retrieval of Content-based 3D Model[J]. Journal of Computer Science, 2004,27(10): 1297-1310.

5. OSADA R, FUNKHOUSER T,et al.Shape distribution[J].ACM

6. Transactions on Graphics, 2002,21(4):807-832.

7. Funkhouser T, Min P, Kazhdan M et al. A search engine for $3 \mathrm{~d}$ models, ACM Transactions on Graphics, 2003, 22(1):83-105.

8. RICHARD J. A survey of free-form object representation and recognition techniques[J]. Computer Vision and Image Understanding, 2001, 81: 166-210.

9. ZHAO Ming-xi.Study on Grid Processing Algorithm Based on Perception Feature [J].Shanghai Jiaotong University,2006.10. 